\title{
A world beyond double-helical nucleic acids: the structural diversity of tetra-stranded G-quadruplexes
}

\author{
Klaus Weisz ${ }^{1}$ (D)
}

Received: 2 July 2021 / Accepted: 13 August 2021 / Published online: 26 August 2021

(c) The Author(s) 2021

\begin{abstract}
Nucleic acids can adopt various secondary structures including double-, triple-, and tetra-stranded helices that differ by the specific hydrogen bond mediated pairing pattern between their nucleobase constituents. Whereas double-helical DNA relies on Watson-Crick base pairing to play a prominent role in storing genetic information, G-quadruplexes are tetra-stranded structures that are formed by the association of guanine bases from G-rich DNA and RNA sequences. During the last few decades, G-quadruplexes have attracted considerable interest after the realization that they form and exert regulatory functions in vivo. In addition, quadruplex architectures have also been recognized as versatile and powerful tools in a growing number of technological applications. To appreciate the astonishing structural diversity of these tetra-stranded structures and to give some insight into basic interactions that govern their folding, this article gives an overview of quadruplex structures and rules associated with the formation of different topologies. A brief discussion will also focus on nonconventional quadruplexes as well as on general principles when targeting quadruplexes with ligands.
\end{abstract}

\section{Graphic abstract}

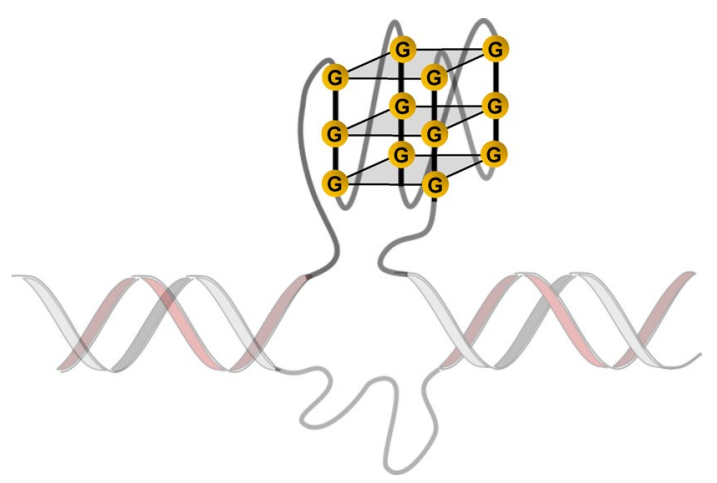

Keywords Nucleic acid · G-quadruplex · G-tetrad · Topology · Glycosidic torsion angle

\section{Introduction}

The basic structural unit of G-quadruplexes was reported as early as 1962 by Gellert, Lipsett, and Davis when they investigated the structure of fibers obtained from a gel of concentrated guanylic acid (GMP) [1]. Their studies pointed

Klaus Weisz

weisz@uni-greifswald.de

1 Institute of Biochemistry, Universität Greifswald, Felix-Hausdorff Str. 4, 17489 Greifswald, Germany to a planar hydrogen-bonded arrangement of four guanine (G) bases related to each other by an axis of fourfold symmetry (Fig. 1a). With a total of eight hydrogen bonds, such an arrangement is expected to be particularly stable and also allows for the formation of linear aggregates upon the stacking of tetrads driven by strong interactions of their large planar surface areas. Yet, the propensity of DNA or RNA oligonucleotides rich in guanine bases for the formation of corresponding G-quadruplexes with a core of stacked guanine tetrads became 
(a)

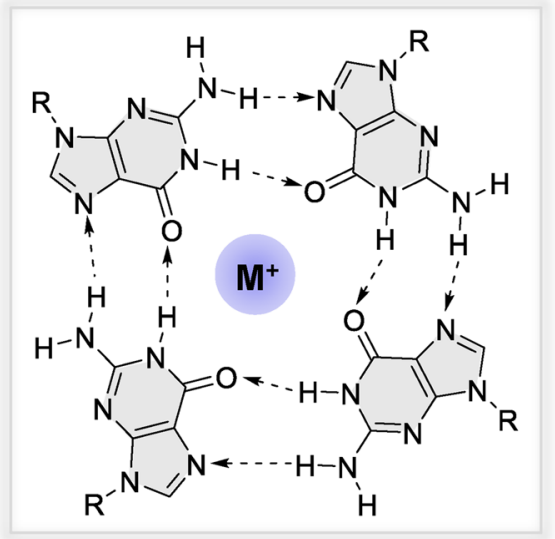

(b)

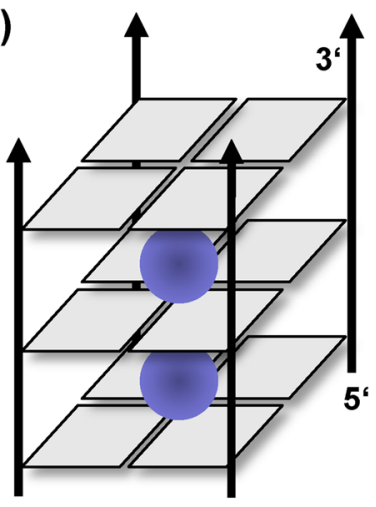

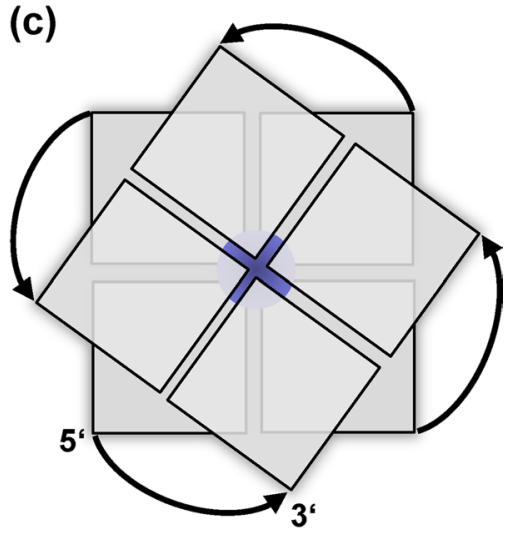

Fig. 1 a G-tetrad with a central metal ion and guanine bases held together by Hoogsteen-type hydrogen bonds; when going from $\mathrm{H}$-bond donor to $\mathrm{H}$-bond acceptor, hydrogen bonds here are progressing in a clockwise direction. b Stack of three G-tetrads with the

apparent only about three decades later when a growing number of high-resolution structures became available.

Due to their potential formation under physiological conditions, studies on G-quadruplexes have surged in past years. However, their formation and existence in the genomes of eukaryotes but also prokaryotes and viruses have only recently been confirmed $[2,3]$. Various biostatistical analyses have estimated between $10^{5}$ and $10^{6}$ putative quadruplex-forming sequences in the human genome [4, 5]. In addition to telomeric sequences, these are frequently found in promoter regions of oncogenic genes. There is little doubt that G-quadruplexes participate and contribute to many physiological processes and also pathological conditions [6, 7]. These include the control of gene expression, the maintenance of genome integrity, cancer progression, and degenerative disorders, making G-quadruplexes attractive targets for therapeutic interventions [8-10]. In addition to their biological function and their significance for medicinal and pharmaceutical strategies, G-quadruplexes have increasingly been employed in various bio- and nanotechnological applications [11-13]. Thus, nucleic acid aptamers and DNAzymes are often based on a G-quadruplex scaffold. Also, various sensor systems for metabolites or metal ions, electronic switches, and nanostructures increasingly rely on the specific properties of G-quadruplexes. Clearly, the conformational variability and unique features of these non-canonical nucleic acids hold a lot of promise for their future exploitation.

\section{G-quadruplex architecture}

A G-tetrad or G-quartet with its square planar arrangement of four guanine bases constitutes the basic structural unit of a G-quadruplex. The Gs are held together by eight Hoogsteen hydrogen bonds involving imino and amino coordination of two metal ions in the central channel between tetrad layers; all four G-columns comprising three consecutive $\mathrm{G}$ residues each are oriented in the same 5'-3' direction. $\mathbf{c}$ Top view of two righthanded helically stacked G-tetrads

hydrogen bond donors at their Watson-Crick edge as well as $\mathrm{O} 6$ and N7 hydrogen bond acceptors at their Hoogsteen edge (Fig. 1a). Depending on a clockwise or anticlockwise orientation of hydrogen bonds when going from donor to acceptor sites, two different tetrad polarities can be distinguished. Typical quadruplexes consist of a G-core comprising two to four G-quartets that are stacked on each other in a helical arrangement; however, three-layered quadruplexes largely predominate (Fig. 1b, c). In such a stacked columnar arrangement, monovalent cations, in particular potassium or sodium ions, are coordinated within the central channel of the G-core that is lined with the guanine carbonyl oxygen atoms. Because of the negative potential within the inner channel, uptake of cations is essential for G-quadruplex stabilization. In general, due to their size and lower free energy of dehydration, potassium ions show a higher stabilizing effect when compared with smaller sodium ions.

Each of the four columns of the G-core comprising two to four guanine bases constitutes a run of consecutive guanosine residues in an oligonucleotide sequence. These G-tracts may either be located on four individual strands, pairwise combined in two strands with a single intervening sequence, or situated within a single strand separated by three intervening sequences to form a tetramolecular, bimolecular, or monomolecular G-quadruplex structure, respectively. In general, G residues within a G-column may be in an anti glycosidic conformation or, as a result of rotating the base around the glycosidic bond toward the sugar moiety, in a syn conformation. However, G-tetrad formation requires all guanine bases of the tetrad to be oriented the same way. Thus, with all G-tracts pointing into the same direction, G nucleotides participating in the tetrad arrangement must adopt the same glycosidic conformation (Fig. 2a). Although both all-anti and all-syn tetrads with opposite tetrad polarity 
are conceivable, all-syn tetrads formed by rotating all four guanine bases are significantly disfavored and only observed in rare cases as a consequence of small syn-anti conformational energy differences of the G nucleotide. By the same reasoning, residues of the same tetrad but located in antiparallel G-tracts, i.e., with opposite 5'-to-3' orientation, must adopt different glycosidic torsion angles to conserve the hydrogen-bonded G-tetrad alignment (Fig. 2b).

In contrast to the constraints imposed on the relative glycosidic conformation of $\mathrm{G}$ residues in each single G-quartet, the pattern of glycosidic bond angles along a G-column is less restricted. These sequential glycosidic conformations determine the polarity of stacked tetrads, resulting in homopolar or heteropolar stacking interactions (Fig. 3). Note, however, that glycosidic conformations along one G-tract determine corresponding conformations of the other three G-tracts in response to their parallel or antiparallel orientation. Because stacking of anti-anti or syn-anti steps is favored over the stacking of anti-syn and syn-syn steps, the preferred syn/anti pattern along the four G-tracts tends to minimize the number of disfavored anti-syn and syn-syn steps [14].

Finally, tetra-stranded quadruplexes feature four grooves separated by the sugar-phosphate backbone of the four G-columns (Fig. 2). These are of equal medium width in the case of four parallel G-columns with the same glycosidic conformation for residues in the same G-tetrad. For an antiparallel G-tract alignment, mixed syn/anti conformations of residues comprising a tetrad will generate different groove dimensions with additional narrow and wide grooves. Following hydrogen bonds from donor to acceptor, residues in an anti $\mathrm{G} \rightarrow$ anti $\mathrm{G}$ or $\operatorname{syn} \mathrm{G} \rightarrow \operatorname{syn} \mathrm{G}$ alignment will form a medium groove, whereas antiG $\rightarrow \operatorname{syn} \mathrm{G}$ and $\operatorname{syn} \mathrm{G} \rightarrow$ anti $\mathrm{G}$ alignments will give rise to wide and narrow grooves, respectively.

\section{G-quadruplex topologies}

Compared with tetramolecular and bimolecular structures, G-quadruplexes formed upon the folding of a single G-rich oligonucleotide have received most attention due to their high relevance not only in biological systems but also in the design of non-natural quadruplex architectures. Typical quadruplex-forming sequences comprise four G-tracts of $\geq 3$ consecutive $G$ residues separated by short intervening sequences $\mathrm{N}_{\mathrm{x}}$. Consequently, conservative search algorithms have been based on a consensus sequence motif $\mathrm{d}\left(\mathrm{G}_{3+} \mathrm{N}_{1-7} \mathrm{G}_{3+} \mathrm{N}_{1-7} \mathrm{G}_{3+} \mathrm{N}_{1-7} \mathrm{G}_{3+}\right)$ for predicting putative quadruplex structures in genomic DNA [15]. Apparently, the four G-columns constituting the quadruplex core are connected by the three intervening sequences that either link two adjacent parallel strands, two adjacent antiparallel strands, or two non-adjacent antiparallel strands to form a propeller (double-chain reversal) loop, a lateral (edge-wise) loop, and a diagonal loop, respectively (Fig. 4). Geometric
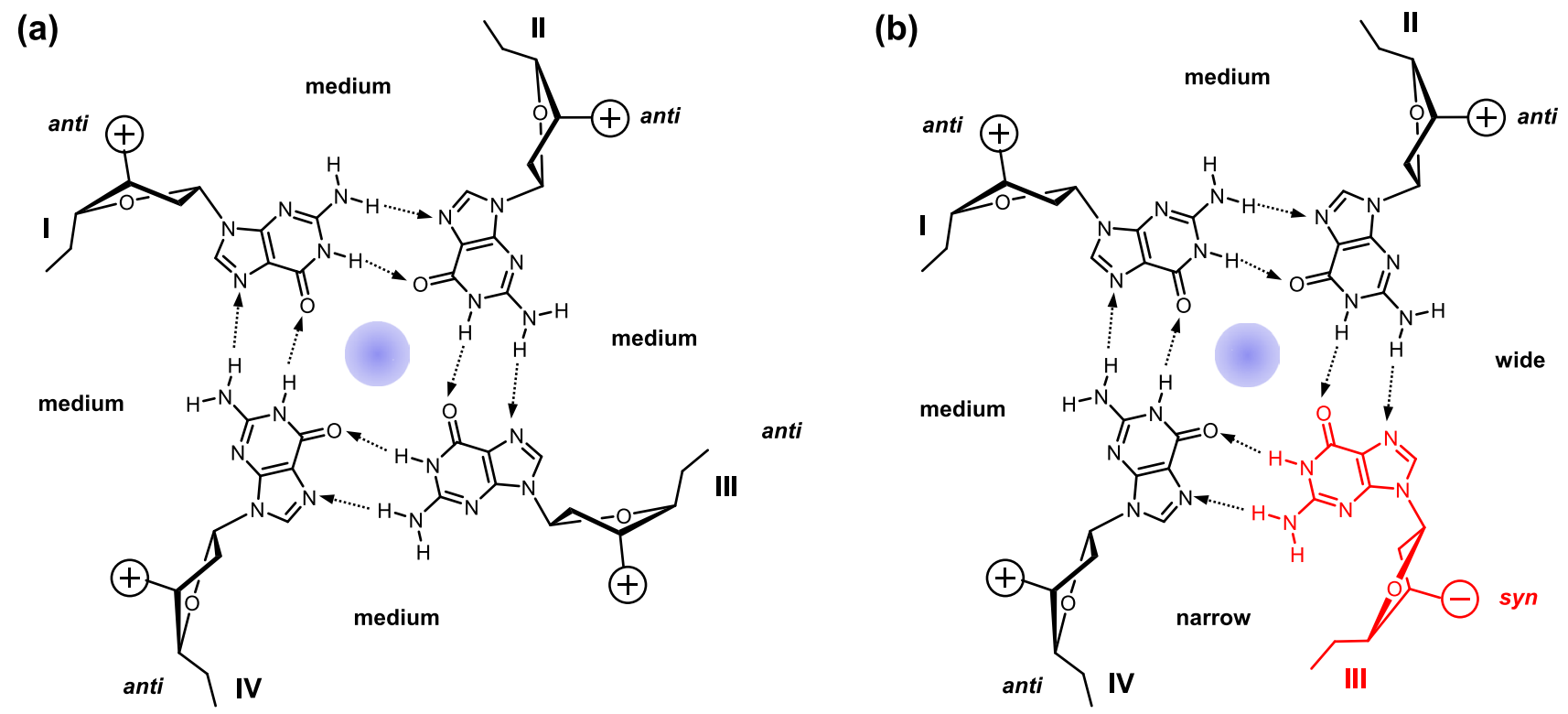

Fig. 2 a G-tetrad with all guanosines in an anti glycosidic torsion angle conformation and all G-columns I-IV with the same $5^{\prime}-3^{\prime}$ backbone orientation ( + , running from top to bottom). b G-tetrad with a flipped antiparallel column III (-, running from bottom to top) with its $\mathrm{G}$ residue adopting a syn conformation to maintain hydrogen

bonding (red). The four grooves are either narrow, medium, or wide, depending on a syn-anti, anti-anti (syn-syn), or anti-syn pattern of adjacent residues within the G-quartet when going from H-bond donor to H-bond acceptor 

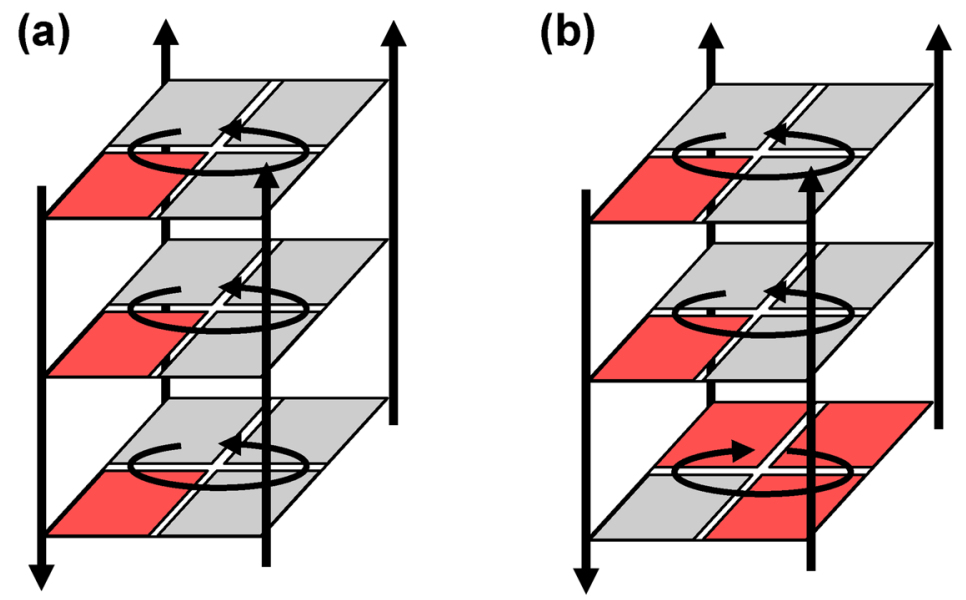

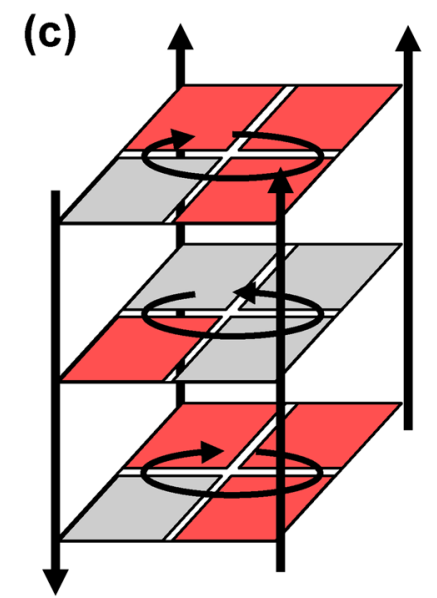

Fig. 3 Three-layered G-quadruplexes with three parallel and one antiparallel G-column and different patterns of glycosidic conforma-

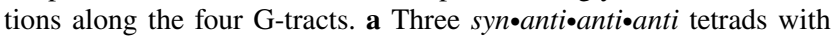
exclusive homopolar stacking formed by the association of three anti-anti-anti and one syn-syn-syn G-tract. b Homopolar as well

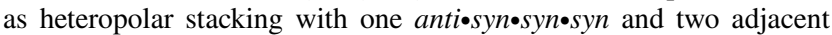
syn•anti•anti•anti tetrads. c Exclusive heteropolar stacking interac-

considerations demand different minimal lengths for the loop-forming segments with typically only a single nucleotide for a most stable propeller loop in a three-tetrad quadruplex, more than one or two nucleotides in the case of lateral loops when bridging a narrow or wide groove, and more than three nucleotides for a diagonal loop bridging distal edges of a G-tetrad.

In theory, there are $3^{3}=27$ possible loop combinations in the presence of three loops, resulting in quadruplexes of different topologies. Additional topologies derive from a clockwise (+) or anticlockwise (-) progression of propeller and lateral loops in a common frame of reference with the 5 '-terminus placed at the lower right corner. It easily becomes apparent that only a fraction of the theoretical folds can be realized for geometric reasons, e.g., topologies with two consecutive diagonal loops are clearly impossible as are two consecutive lateral loops running clockwise and counter-clockwise. For characterizing and discriminating the various folds, a simple descriptor composed of the type and progression of consecutive loops may be used [16, 17]. Thus, abbreviating lateral, propeller, and diagonal loops with "l," "p," and "d," respectively, the designation (+ld -p) describes a quadruplex topology with a first lateral loop at the 5 '-end running clockwise, a central diagonal loop, and a third propeller loop at the 3 '-end running counter-clockwise. Notably, whereas extended geometric descriptors can be used, the simple description of quadruplex topologies as introduced above does not include information on the number of G-tetrads and the geometry of grooves, nor does it discriminate among different patterns of glycosidic bond angles along the individual G-columns.

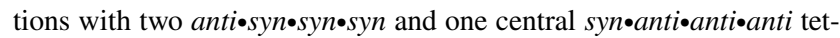
rad. Note that sequential glycosidic conformations along one G-tract determine conformations of the other three G-columns for given strand polarities. Based on the number of (dis)favored steps, the conformation in $\mathbf{b}$ tends to be preferred. Cyclic arrows indicate tetrad polarities, whereas gray and red rectangles represent anti and syn $\mathrm{G}$ residues, respectively
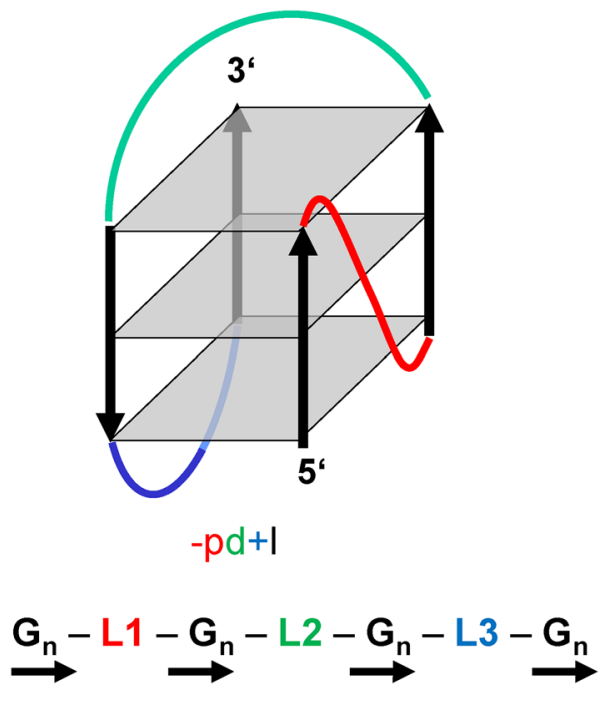

Fig. 4 General sequence of an intramolecular G-quadruplex (bottom) and schematic representation of its folding into a three-layered G-quadruplex $(n=3)$ with a topology described by $(-p d+1)$; the first intervening sequence L1 forms a propeller loop running counterclockwise $(-p$, red) whereas L2 forms a diagonal (d, green) and L3 a lateral loop running clockwise $(+1$, blue)

There are three major families of conventional G-quadruplexes (Fig. 5). A parallel topology as almost exclusively observed for RNA quadruplexes is based on four G-tracts oriented in the same direction. Here, all $\mathrm{G}$ residues of the G-core adopt the same glycosidic conformation, being anti in nearly all cases. G-quadruplexes of a $(3+1)$ hybrid-type comprise one antiparallel and three parallel G-columns, 
frequently realized by $(-p-1-1)$ and $(-1-1-p)$ topologies termed hybrid-1 and hybrid-2, respectively. Finally, quadruplexes with an antiparallel topology feature pairs of parallel and antiparallel G-columns. Here, a G-column may have two adjacent antiparallel G-tracts as in the chairlike $(+1+1+1)$ topology or both a parallel and an antiparallel neighboring column as in the basket-type $(-1 d+1)$ topology. (a)

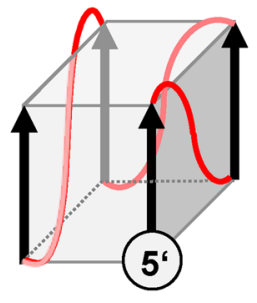

$-p-p-p$

parallel

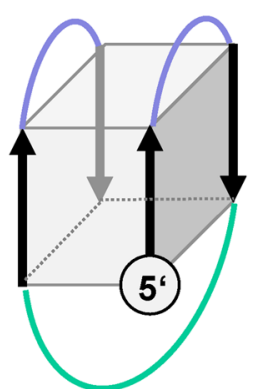

$-\mid d+1$

(b)

antiparallel, ,basket' form

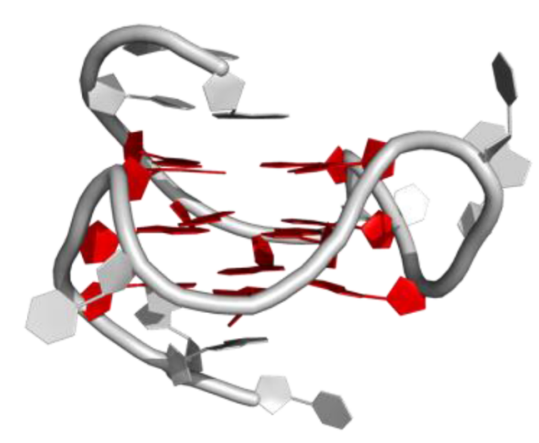

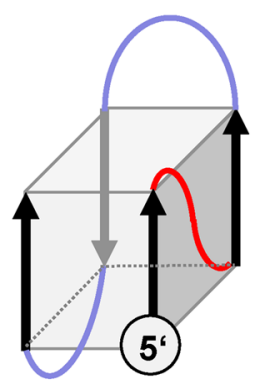

$-\mathrm{p}-\mathrm{I}-\mathrm{I}$

$(3+1)$ hybrid-1

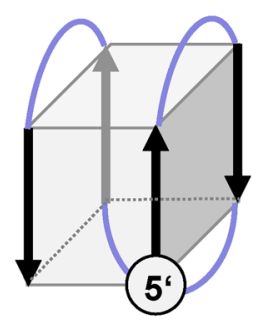

$-|-|-\mid$

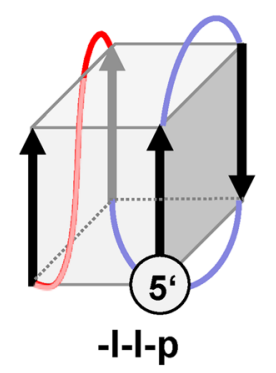

$(3+1)$ hybrid-2

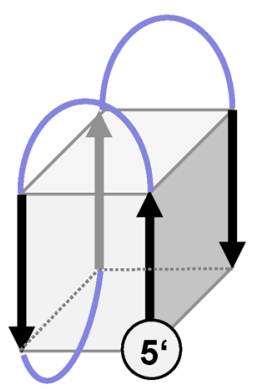

$++1+1$

antiparallel, ,chair' form
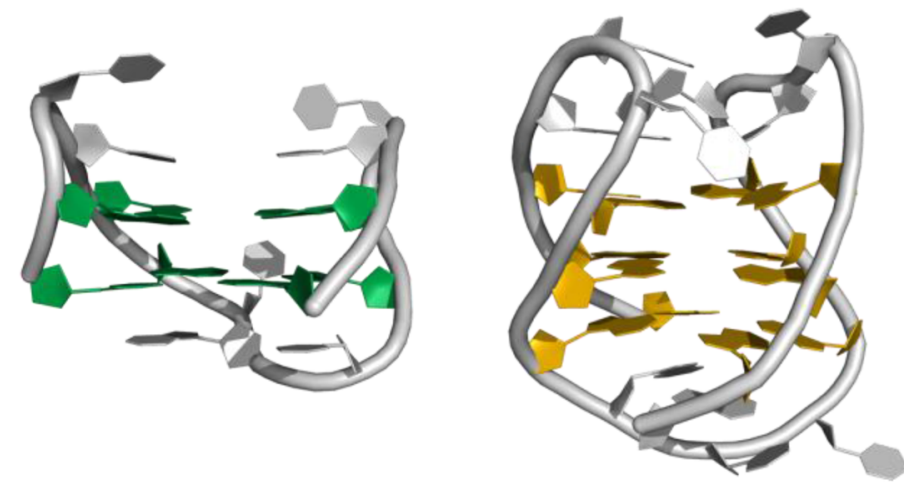

Fig. 5 a Schematic representation of different G-quadruplex topologies and their notation for loop progression. Note that the two antiparallel chair-type folds $(-1-1-1)$ and $(+1+1+1)$ are topologically different. b Representative three-dimensional solution structures. Three-tetrad parallel quadruplex $(-p-p-p)$ with three propeller loops (left, core residues shown in red, pdb ID: 1XAV); two-tetrad chair-type quadruplex $(+1+1+1)$ with three lateral loops (center, core residues shown in green, pdb ID: 2LYG); three-tetrad basket-type quadruplex $(-1 d+1)$ with two lateral and one diagonal loop (right, core residues shown in yellow, pdb ID: 143D) 


\section{Stability and polymorphism of G-quadruplexes}

From an energetic point of view, the thermodynamic stability of G-quadruplexes mostly derives from the stacking of tetrads with the coordination of cations. However, loop domains as well as $5^{\prime}$ - and $3^{\prime}$-flanking sequences may significantly contribute to the quadruplex stability by tertiary interactions, often forming an additional capping structure on an outer G-tetrad. Capping structures or a dimerization by end-stacking are frequently observed phenomena and particularly important for adding stability to quadruplex scaffolds comprising only two tetrad layers [18].

In addition to overhang sequences, the type of coordinated cation as well as dehydrating effects from molecular crowding may significantly influence the G-quadruplex stability but also the folding pathway of a G-rich sequence. Compared with sodium ions, potassium ions are not only more stabilizing but also tend to promote folding into parallel topologies. Typically, parallel quadruplexes with short one-nucleotide propeller loops exhibit a considerable thermal stability, with melting temperatures often exceeding $90{ }^{\circ} \mathrm{C}$ in $120 \mathrm{mM} \mathrm{K}^{+}$solutions. Various quadruplex topologies are often close in their thermodynamic stability and coexist in solution. Consequently, small changes in the flanking sequence or in outer conditions can easily shift folding into another major topology.
Being a most prominent example, the human telomeric sequence d[GGG(TTAGGG) $)_{3}$ ] may adopt five different intramolecular G-quadruplex topologies under different experimental conditions (Fig. 6). Comprising a 5'-A flanking residue, it adopts an antiparallel basket-type G-quadruplex with a core of three stacked G-tetrads in a $\mathrm{Na}^{+}$solution. With two pairs of anti-syn-anti and syn-anti-syn G-columns, it only features heteropolar tetrad stacking interactions. In a $\mathrm{K}^{+}$-containing crystal, the same sequence folds into a parallel G-quadruplex with three propeller loops, allanti $\mathrm{G}$ residues, and exclusive homopolar tetrad stacking. Being in a $\mathrm{K}^{+}$solution, two $(3+1)$ hybrid conformations, namely $(3+1)$ hybrid- 1 and $(3+1)$ hybrid- 2 have been identified for d[TAGGG(TTAGGG) $)_{3}$ ] and d[TAGGG(TTA GGG) ${ }_{3} \mathrm{TT}$ ], respectively. Both structures have one propeller and two lateral loops but differ in their loop arrangement. With three syn-anti-anti and one syn-syn-anti G-column, tetrads interact through homopolar as well as heteropolar stacking. Finally, an antiparallel basket-type G-quadruplex termed hybrid Form 3 comprising only two G-tetrad layers of opposite polarity and with a third G of the four G-runs shifted into loop segments was formed by the human telomeric sequence with a $3^{\prime}-\mathrm{T}$ flanking residue in $\mathrm{K}^{+}$solution. Here, extensive base pairing and stacking in the loops capping the G-tetrad core contribute to the ability of this twolayered structure to successfully compete with a three-tetrad architecture [19].
Fig. 6 Topologies of G-quadruplexes formed by the human telomeric sequence. a Antiparallel basket-type quadruplex $(-1 \mathrm{~d}+1)$ observed for d[A(GGGTTA $\left.)_{3} \mathrm{GGG}\right]$ in a $\mathrm{Na}^{+}$solution. b Parallel quadruplex $(-p-p-p)$ formed by d[A(GGGTTA) $)_{3}$ GGG] in a $\mathrm{K}^{+}$-containing crystal. $\mathbf{c}(3+1)$ hybrid-1 $(-\mathrm{p}-1-1)$ observed for d[TA(GGGTTA) ${ }_{3}$ GGG] in a $\mathrm{K}^{+}$solution. $\mathbf{d}(3+1)$ hybrid-2 $(-1-1-\mathrm{p})$ observed for d[TA(GGGTTA) ${ }_{3}$ GGGTT] in a $\mathrm{K}^{+}$solution. e Antiparallel basket-type quadruplex $(-1 d+1)$ with only two G-tetrad layers (Form 3 ) formed by d[(GGG TTA) $)_{3}$ GGGT] in $\mathrm{K}^{+}$solution. Anti and syn guanines are colored gray and red, respectively (a)

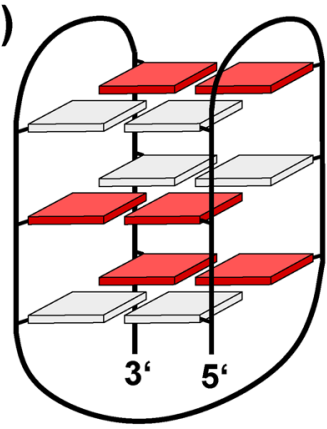

(b)

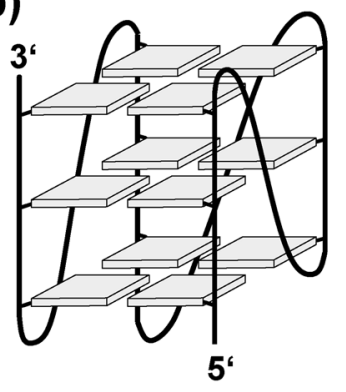

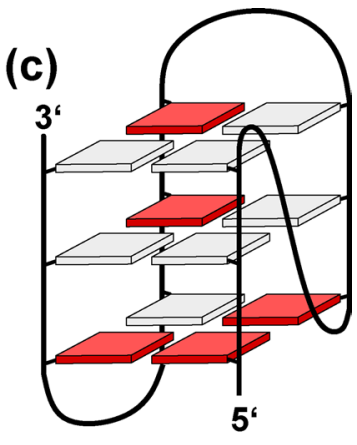

(d)

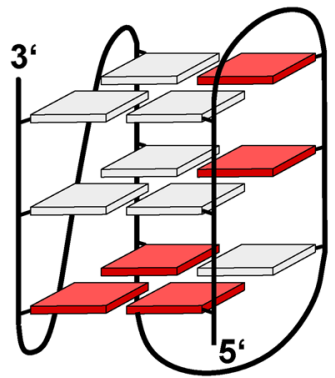

(e)

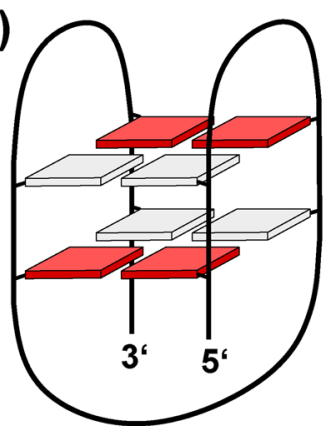




\section{Nonconventional G-quadruplexes with broken G-columns}

The structural diversity of G-quadruplexes further increases due to their ability to compensate for guanine deficiencies and to fold into nonconventional quadruplex structures with broken G-columns comprising noncontiguous Gs [20]. Thus, intramolecular three-tetrad quadruplexes may even form from sequences lacking four tracts of $\geq 3$ consecutive guanosines, contradicting the typical consensus sequence. With a single G-run comprising only two G residues, a vacant site in an outer tetrad of the folded species may be occupied by an external guanine derivative present in solution. Filling of an empty $\mathrm{G}$ position can also be realized by an unusual backbone progression. Such a fold will depend on the particular G-rich sequence and result in quadruplexes with an interrupted G-tract. Short bulges protruding within a G-column are often tolerated in case of a split G-run, albeit compromising the quadruplex stability. In addition to the formation of intertwined or interlocked dimeric and multimeric structures, other unusual structural motifs allowing for stable quadruplex formation in the presence of a shortened G-tract include snapback and V-shaped loops (Fig. 7). Clearly, this variability in arriving at a thermodynamically stable fold combined with additional stabilizing interactions of loop and overhang residues represents a challenge for the sequence-based design and prediction of favored G-quadruplex topologies.

\section{Quadruplex-ligand interactions}

To date, a plethora of quadruplex-binding ligands have been designed and tested, also including macrocyclic and metalloorganic compounds $[21,22]$. Interest in quadruplex binders was greatly stimulated by their potential use as novel anticancer agents. Such use is mostly based on their ability to induce and stabilize quadruplexes at G-rich promoter regions of oncogenes and at telomeric ends, interfering with gene expression through the cellular transcription machinery and with telomerase-mediated telomere elongation in cancer cells. In fact, some quadruplex-binding ligands have been shown to be effective in targeting quadruplex-forming regions in vivo and to inhibit cancer cell growth. The quadruplex interacting drug quarfloxin is the first candidate to have entered phase II clinical trials for the treatment of neuroendocrine carcinomas [23].

A major issue when developing quadruplex ligands is their specificity and discriminating power for quadruplex structures in the presence of predominating double-helical DNA. Thus, the majority of quadruplex ligands features a flat polycyclic ring system with a large aromatic surface area to optimize stacking on an outer G-quartet of the quadruplex. As a consequence, binding largely depends on strong $\pi-\pi$ stacking interactions. Intercalation between tetrads as frequently observed with duplex DNA seems to be disfavored due to the energetic penalty for unwinding a tetra-stranded structure and for expulsion of the centrally coordinated metal ions. Overhang sequences, often forming a binding pocket, and additional interactions between side chains attached to the ligand aromatic core with the quadruplex backbone, loops, or inside quadruplex grooves may enforce binding and open up possibilities to selectively target particular quadruplex structures with minimal off-target effects (Fig. 8). Thus, quadruplex grooves with different geometry support more selectivity in binding, but in contrast to duplex DNA, only few ligands were demonstrated to exclusively interact within a quadruplex groove. Ongoing attempts for achieving better selectivity among quadruplexes by adding more specific interactions in addition to tetrad stacking are challenging but will ultimately offer great promise for in vivo applications of corresponding ligands.
Fig. 7 Schematic representation of nonconventional three-layered G-quadruplexes featuring a broken G-column with only two consecutive guanosines (marked by the orange section of the backbone); a snapback loop (left) and a V-shaped loop (right) fill a vacant position of an outer tetrad (colored turquoise)
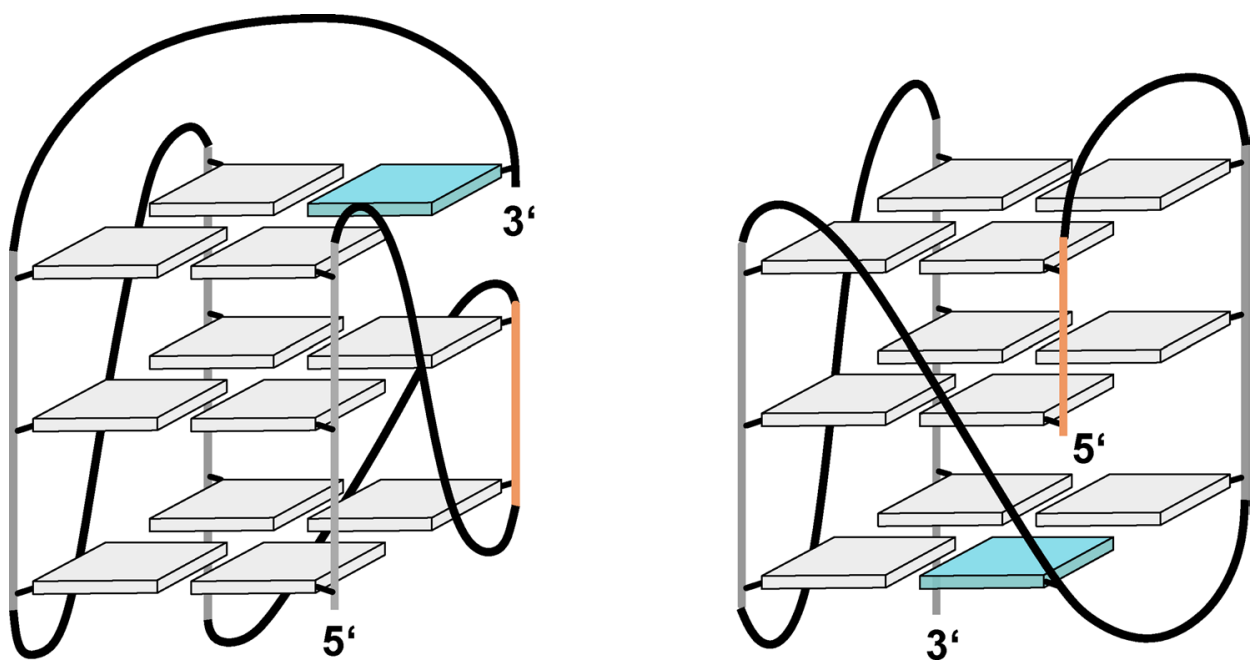

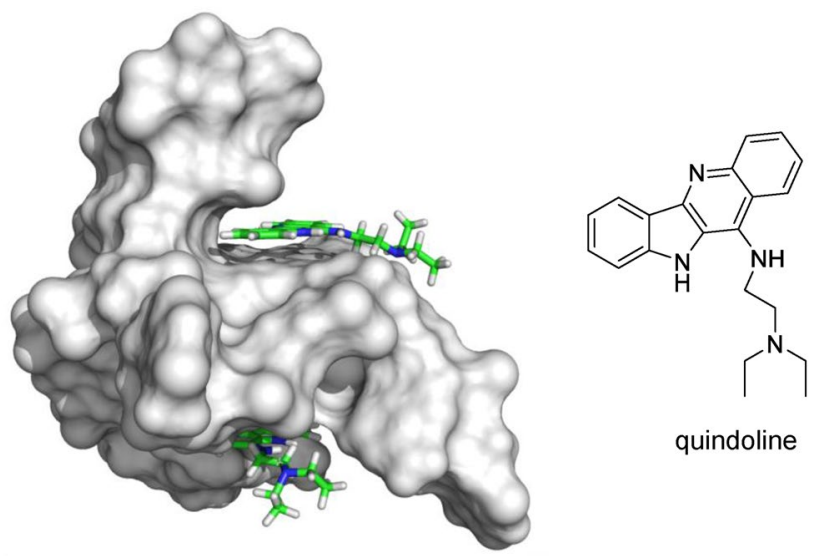

quindoline

Fig. 8 External stacking of a quindoline ligand to both outer faces of a parallel quadruplex shown as a space filling model in side view; extending 5'- and 3'-overhang sequences on top and bottom form a binding pocket for the ligand (pdb ID: $2 \mathrm{~L} 7 \mathrm{~V}$ )

\section{Conclusions}

G-quadruplexes have become one of the most intensely studied nucleic acid structures over the past two decades. Their existence across all organisms and biological role in vivo have sparked interest for these remarkable structures in biology as well as in medicine and pharmacology. On the other hand, G-quadruplexes have been shown to adopt an amazing number of different topologies, and their specific properties make them promising tools for various technological applications. We are currently seeing a growing understanding of critical interactions within G-quadruplex architectures. Numerous structural and thermodynamic studies on quadruplexes have yielded geometric formalisms for a description of their folds, and empirical rules have emerged for the propensity of G-rich sequences to favor a particular conformer in a given environment. Current efforts aim at a rational sequence-based design of quadruplex topologies and of high-affinity ligands interacting with quadruplexes through highly specific interactions. As a result, investigations on G-quadruplexes are anticipated to continue playing a growing role in many diverse areas of basic and applied science.

Funding Open Access funding enabled and organized by Projekt DEAL.

\section{Declarations}

Funding Supported by the Deutsche Forschungsgemeinschaft (grant no. WE 1933/15-1).

Conflict of interest The authors declare no conflict of interest.

Data availability Not applicable for that section.
Author contributions Not applicable for that section.

Code availability Not applicable for that section.

Ethics approval Not applicable for that section.

Consent to participate Not applicable for that section.

Consent for publication Not applicable for that section.

Open Access This article is licensed under a Creative Commons Attribution 4.0 International License, which permits use, sharing, adaptation, distribution and reproduction in any medium or format, as long as you give appropriate credit to the original author(s) and the source, provide a link to the Creative Commons licence, and indicate if changes were made. The images or other third party material in this article are included in the article's Creative Commons licence, unless indicated otherwise in a credit line to the material. If material is not included in the article's Creative Commons licence and your intended use is not permitted by statutory regulation or exceeds the permitted use, you will need to obtain permission directly from the copyright holder. To view a copy of this licence, visit http://creativecommons.org/licenses/by/4.0/.

\section{References}

1. Gellert M, Lipsett MN, Davies DR (1962) Helix formation by guanylic acid. Proc Natl Acad Sci U S A 48:2013-2018

2. Lam EYN, Beraldi D, Tannahill D, Balasubramanian S (2013) G-quadruplex structures are stable and detectable in human genomic DNA. Nat Commun 4:1796

3. Laguerre A, Hukezalie K, Winckler P, Katranji F, Chanteloup G, Pirrotta M, Perrier-Cornet J-M, Wong JMY, Monchaud D (2015) Visualization of RNA-quadruplexes in live cells. J Am Chem Soc 137:8521-8525

4. Todd AK, Johnston M, Neidle S (2005) Highly prevalent putative quadruplex sequence motifs in human DNA. Nucleic Acids Res 33:2901-2907

5. Chambers VS, Marsico G, Boutell JM, Di Antonio M, Smith GP, Balasubramanian S (2015) High-throughput sequencing of DNA G-quadruplex structures in the human genome. Nat Biotechnol $33: 877-881$

6. Hänsel-Hertsch R, Di Antonio M, Balasubramanian S (2017) DNA G-quadruplexes in the human genome: detection, functions and therapeutic potential. Nat Rev Mol Cell Biol 18:279-284

7. Varshney D, Spiegel J, Zyner K, Tannahill D, Balasubramanian S (2020) The regulation and functions of DNA and RNA G-quadruplexes. Nat Rev Mol Cell Biol 21:459-474

8. Neidle S (2017) Quadruplex nucleic acids as targets for anticancer therapeutics. Nat Rev Chem 1:0041

9. Balasubramanian S, Hurley LH, Neidle S (2011) Targeting G-quadruplexes in gene promoters: a novel anticancer strategy? Nat Rev Drug Discov 10:261-275

10. Müller S, Rodriguez R (2014) G-quadruplex interacting small molecules and drugs: from bench toward bedside. Expert Rev Clin Pharmacol 7:663-679

11. Neo JL, Kamaladasan K, Uttamchandani M (2012) G-quadruplex based probes for visual detection and sensing. Curr Pharm Des 18:2048-2057

12. Chiorcea-Paquim A-M, Eritja R, Oliveira-Brett AM (2018) Electrochemical and AFM characterization of G-quadruplex electrochemical biosensors and applications. J Nucleic Acids 2018:1-20

13. Mergny JL, Sen D (2019) DNA quadruple helices in nanotechnology. Chem Rev 119:6290-6325 
14. Cang X, Šponer J, Cheatham TE III (2011) Explaining the varied glycosidic conformational, G-tract length and sequence preferences for anti-parallel G-quadruplexes. Nucleic Acids Res 39:4499-4512

15. Huppert JL, Balasubramanian S (2005) Prevalence of quadruplexes in the human genome. Nucleic Acids Res 33:2908-2916

16. Webba da Silva M (2007) Geometric formalism for DNA quadruplex folding. Chem-A Eur J 13:9738-9745

17. Karsisiotis AI, O'Kane C, Webba da Silva M (2013) DNA quadruplex folding formalism-a tutorial on quadruplex topologies. Methods 64:28-35

18. Jana J, Weisz K (2021) Thermodynamic stability of G-quadruplexes: impact of sequence and environment. ChemBioChem. https://doi.org/10.1002/cbic.202100127

19. Lim KW, Amrane S, Bouaziz S, Xu W, Mu Y, Patel DJ, Luu KN, Phan AT (2009) Structure of the human telomere in $\mathrm{K}^{+}$solution: a stable basket-type G-quadruplex with only two G-tetrad layers. J Am Chem Soc 131:4301-4309

20. Jana J, Mohr S, Vianney YM, Weisz K (2021) Structural motifs and intramolecular interactions in non-canonical G-quadruplexes. RSC Chem Biol 2:338-353

21. Monchaud D, Teulade-Fichou MP (2008) A hitchhiker's guide to G-quadruplex ligands. Org Biomol Chem 6:627-636

22. Zhang S, Wu Y, Zhang W (2014) G-quadruplex structures and their interaction diversity with ligands. ChemMedChem 9:899-911

23. Balaratnam S, Schneekloth JS Jr (2020) Transcriptional regulation of MYC through G-quadruplex structures. Annu Rep Med Chem 54:361-407

Publisher's Note Springer Nature remains neutral with regard to jurisdictional claims in published maps and institutional affiliations. 